\title{
Azygos anterior cerebral artery aneurysm with concomittant vascular anomaly: Case report
}

\author{
Özcan Binatli $^{1}$, Füsun Demirçivi Özer ${ }^{1}$, Murat Aydin $^{2 *}$, Ebru Çiçek ${ }^{3}$, Yiğit Can Binatli ${ }^{1}$ \\ ${ }^{1}$ Department of Neurosurgery, Tepecik Educational and Research Hospital, İzmir, Turkey \\ ${ }^{2}$ Department of Neurosurgery, Karaman State Hospital, Karaman, Turkey \\ ${ }^{3}$ Departement of Radiology, Tepecik Educational and Research Hospital, İzmir, Turkey \\ Email: maydin73@gmail.com, aobinatli@hotmail.com, fdcviozer@hotmail.com, cicekebru2000@yahoo.com, \\ aobinatli@hotmail.com
}

Received 25 May 2012; revised 29 October 2012; accepted 30 November 2012

\begin{abstract}
A 51-year-old man presented with subarachnoid haemorrhage with mild hydrocephaly. Digital substraction angiography and 3-D Computerised Angiogram (CT) revealed a saccular aneurysm at bifurcation of azygos anterior cerebral artery (ACA) and other vascular variations such as vertebral artery fenestration and hypoplasia in one anterior cerebral artery. We performed aneurysmal neck clipping with good outcome and postoperative 3-D CT angiogram showed complet obliteration of aneurysm. Although azygos arteries are rare in healthy population, aneurysms of azygos ASA are not rare due to increased haemodynamic stress. We wanted to point out to better visualisation of anatomical variations at 3-D CT angiogram comparing DSA in patients with intracranial aneurysm.
\end{abstract}

Keywords: Azygos Anterior Cerebral Artery; Aneurysm; Anomaly

\section{INTRODUCTION}

Unpaired distal anterior cerebral artery (ACA), also known as azygos ACA, is a rare condition in anatomical and angiographic studies. Perlmutter and Rhoton reported one case in 25 adult cadaveric brains [1]. Stefani et al. also noted one case in 38 cadaveric brains [2]. It is not surprising that this kind of anatomical variation may predispose individuals to aneurysm formation, due to alterations in hemodynamics.

Indeed, reports on saccular aneurysm coupled with azygos ACA are more frequent than previously estimated. Its occurance rate has been reported to be between 13\% and $71 \%$ [3-7]. Other intracranial malformations, such as agenesis of the corpus callosum, hydranencephaly, and vascular abnormalities, have drawn attention in nearly all

${ }^{*}$ Corresponding author. published reports [7-10].

In this paper, we report a patient with a subarachnoid hemorrhage from a saccular aneurysm on azygos ACA associated with a microaneurysm at the middle cerebral artery bifurcation and a fenestration at the vertebrobasilar junction detected by 3D CT angiography.

\section{CASE REPORT}

A 51-year-old man presented with a sudden headache and neck stiffness. CT imaging revealed a subarachnoid hemorrhage in the basal cisterns and an interhemispheric fissure with a small hematoma near the same fissure (Figure 1) Mild ventriculomegaly was detected. Conventional cerebral angiography showed hypoplasia at the left A1, an azygos ACA with saccular aneurysm at the bifurcation. A 3-D CT angiogram revealed a more sophisticated vascular network, such as azygos ACA with aneurysm, another aneurysm at the middle cerebral artery bifurcation, and a fenestration at the vertebrobasilar junction (Figure 2).

A right paramedian frontal craniotomy was performed

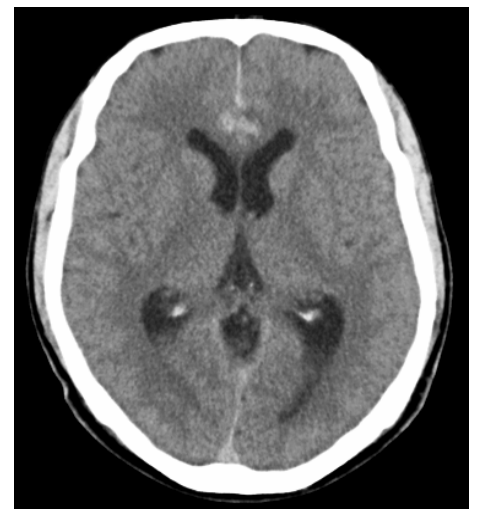

Figure 1. Posthemorrhage CT scan revealing subarachnoid hemorrhage and a small hematoma in interhemispheric fissure. 


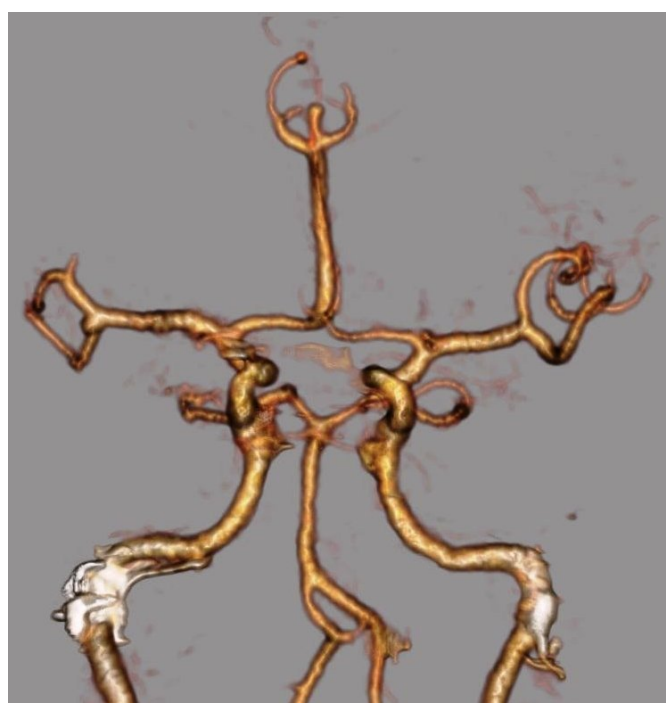

Figure 2. 3D CT angiogram demonstrating azygos ACA and aneurysm, another microaneurysm at the middle cerebral artery bifurcation and fenestration at the vertebrobasilar junction on the same image.

to allow for an interhemispheric approach on the third day of hemorrhage. Azygos ACA and its branches were seen in the corpus callosum. The fundus of the aneurysm was buried in neighboring cingulate gyrus. Following proximal and distal control of arteries, dissection of the neck of the aneurysm was performed and a curved clip was applied. The postoperative course was uneventful. The ventriculomegaly regressed, and the patient was discharged 7 days later after the operation without incident. One month later, 3-D CT angiography demonstrated patency of vessels with complete obliteration of the aneurysm (Figure 3).

\section{DISCUSSION}

In 18-mm embryos (40 days) the anterior cerebral artery takes a medial course. Four days later, the arteria communicans anterior develops from the anterior plexus. Anterior cerebral arteries run in a rostro-occipital direction with the development of the corpus callosum [11]. The unusual fusion of paired post-communicant segments of ACA originates either from the medial branch of the olfactory artery at the initial 16-mm stage of embryogenesis or the persistance of the median artery in the corpus callosum at the $20-24 \mathrm{~mm}$ stage $[2,4]$.

Although azygos ACA has a low incidence (1\% - 4\%) in the adult population or cadaveric series $[1,2]$, it is not rare within subarachnoid hemorrhage cases. Except for the report of Hernesniemi et al. [12], nearly all series on distal ACA aneurysm noted some cases with subarachnoid hemorrhage from the rupture of an azygos ACA aneurysm [5-7]. This may be explained by the susceptibility of aneurysm formation where blood flow has dou-

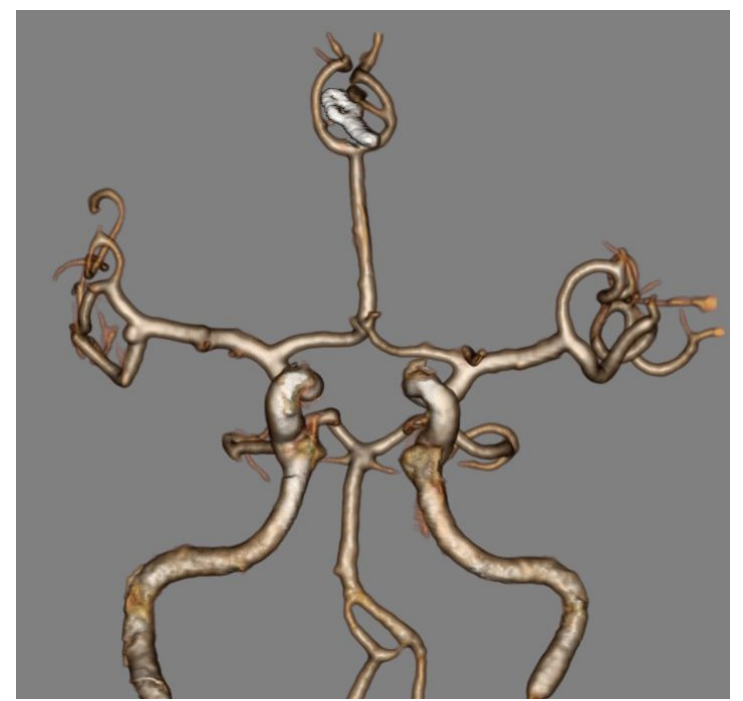

Figure 3. Postoperative 3D CT angiogram indicating complete obliteration of the aneurysm by clipping of the patent parent arteries.

bled and the corresponding hemodynamic pressure has increased in place of normally paired distal ACA [4].

As a variant of normal embryogenesis, azygos ACA accompanies other vascular or nonvascular malformations, such as arteriovenous malformation, aneurysm, fenestration, corpus callosum agenesis, and hydranancephaly [7-10]. The existence of an aneurysm was considered to be a congenital defect in the tunica media, and hemodynamic stress applied in this area can produce aneurysm formation and growth [4].

Treatment of aneurysms of azygos ACA supposes more risky comparisons with paired distal ACA [13]. Narrowing or kinking of unpaired arteries may result in ischemia in both frontal lobes. Consequently, clip application and control of the parent arteries must be done meticulously. In our case, multiple vascular anomalies were detected in both conventional and 3-D CT angiography; 3-D CT angiography was more helpful for determining vascular architecture. No other anomaly was seen in magnetic resonance imaging taken during the postoperative period. The operation and post-operative courses were uneventful.

\section{CONCLUSION}

Although the incidence of azygos ACA is rare in the normal population, aneurysm formation in azygos ACA is frequent with both associated congenital vascular anomalies and high vascular hemodynamics. 3-D CT angiographic examination reveals all anatomical variations, as well as digital substruction for angiographic investigations. Clipping of azygos artery aneurysms is more challenging than paired distal ACA aneurysms, because of the bi-hemispheric nature of the vascular supply of the 
unpaired parent artery.

\section{REFERENCES}

[1] Perlmutter, D. and Rhoton Jr., A.L. (1978) Microsurgical anatomy of the distal anterior cerebral artery. Journal of Neurosurgery, 49, 204-228. doi:10.3171/jns.1978.49.2.0204

[2] Stefani, M.A., Schnaider, F.L., Marrone, A.C.H., Severino, A.G., Jackowsky, A.P. and Wallace, M.C. (2000) Anatomic variations of anterior cerebral artery cortical branches. Clinical Anatomy, 13, 231-236. doi:10.1002/1098-2353(2000)13:4<231::AID-CA1>3.0.C $\underline{\mathrm{O} ; 2-\mathrm{T}}$

[3] Niizuma, K., Kwak, R., Uchida, K. and Suzuki, J. (1981) Aneurysms of the azygos anterior cerebral artery. Surgical Neurology, 15, 225-228. doi:10.1016/0090-3019(81)90150-6

[4] Huh, J.S., Park, S.K., Skin, J.J. and Kim, T.H. (2009) Saccular aneurysms of the azygos anterior cerebral artery: Three case reports. Journal of Korean Neurosurgical Society, 42, 342-345. doi:10.3340/jkns.2007.42.4.342

[5] Yaşargil, M.G. (1984) Microneurosurgery. Georg Thiema Verlag, New York.

[6] Lee, J.W., Lee, K.C., Kim, Y.B. and Huh, S.K. (2008) Surgery for distal anterior cerebral artery aneurysms. Surgical Neurology, 70, 153-159. doi:10.1016/j.surneu.2008.03.012
[7] Eom, K.S. and Kang, S.D. (2001) A2 anomalies associated with anterior cerebral artery aneurysms. Korean Journal of Cerebrovascular Disease, 3, 159-162.

[8] Auguste, K.I., Vare, M.L. and Lawton, M.T. (2004) Nonsaccular aneurysms of the azygos anterior cerebral artery. Neurosurgical Focus, 17, E12.

[9] Jagetia, A., Kumar, P.N., Sinha, S. and Sharma, A. (2007) Saccular bilobed aneurysm of an azygos anterior cerebral artery. Journal of Clinical Neurology, 14, 777-779. doi:10.1016/j.jocn.2006.02.005

[10] Kobayashi, S., Yuge, T., Sugita, Y., et al. (1986) Azygos anterior cerebral artery aneurysm associated with fenestration of the anterior cerebral artery. The Kurume Medical Journal, 33, 149-153. doi:10.2739/kurumemedj.33.149

[11] Lang, J. (1995) Skull base and related structures. Schattauer, New York.

[12] Hernesniemi, J., Tapaninaho, A., Vapalahti, M., Niskanen, M., Kari, A. and Luukkonen, M. (1992) Saccular aneurysms of the distal anterior cerebral artery and its branches. Neurosurgery, 31, 994-999. doi:10.1227/00006123-199212000-00002

[13] Park, J. and Kan, D.H. (2009) In situ rescue bypass for iatrogenic avulsion of parent artery during clipping large pericallosal artery aneurysm. Journal of Korean Neurosurgical Society, 46, 68-70. doi:10.3340/jkns.2009.46.1.68 